\title{
New Stratified Bayesian Estimators Using Warner's Randomized Response Technique Through Mixed Priors
}

\author{
A. O. Adepetun ${ }^{1 *}$, A. A. Adewara ${ }^{2}$ \\ ${ }^{1}$ Department of Statistics, Federal University of Technology, PMB 704, Akure, Ondo State, Nigeria \\ ${ }^{2}$ Department of Statistics, University of Ilorin, PMB 1515, Ilorin, Kwara State, Nigeria
}

Received 2 January 2018, accepted in final revised form 15 May 2018

\begin{abstract}
In this paper, we propose new stratified Bayesian estimators of population proportion of a sensitive trait by adopting a mixture of alternative beta distributions as quantification of prior information in a stratified random sampling situation. Data were collected through Warner's randomized response technique. To study the performance of the newly developed stratified estimators, mean squared error and absolute bias were used as performance criteria. The proposed estimators were compared with the existing one. We observed that the proposed estimators are more sensitive to responses than the existing one at various sample sizes respectively.
\end{abstract}

Keywords: Stratified Bayesian estimators; Mixed priors; Population proportion; Randomized response technique; Sensitive trait.

(C) 2018 JSR Publications. ISSN: 2070-0237 (Print); 2070-0245 (Online). All rights reserved.

doi: http://dx.doi.org/10.3329/jsr.v10i3.35174 J. Sci. Res. 10 (3), 249-259 (2018)

\section{Introduction}

Gathering information about a sensitive trait such as induced abortion, drug usage, tax evasion, examination malpractices etc. rampant in a human population is a complicated issue. Questioning directly from the respondents about exhibition of a stigmatized trait generally leads to doctoring of the true answers. This may be due to fear of social stigma or counter-attacks. However, information about the prevalence of such characteristic in the population is essential due to many socioeconomic reasons. To tackle this issue, Warner [1] introduced a clever technique of survey to gather information about sensitive characteristic by ensuring confidentiality and anonymity to the respondents. To date, many developments and variants of Warner's Randomized Response Technique were developed by different researchers [2-8]. Interested readers may also see the articles [912] for some more literatures on the topic. Once information on the sensitive characteristic(s) have been gathered using any of the cited techniques above, we then

\footnotetext{
* Corresponding author: aoadepetun@ futa.edu.ng
} 
proceed to the estimation stage in which we provide estimates of the corresponding population characteristic(s) based on our sample data. For this singular reason, we have classical framework which is quite popular but the Bayesian technique in this regard is inevitable as it takes care of the situations where parameter uncertainties are involved. This uncertainty is quantified in the form of a prior distribution which is further updated using our sample data for the said estimation purposes.

To make the Bayes estimator performs well, there is need to incorporate stratified random sampling technique into the Bayesian estimation framework. When researchers hold different beliefs in terms of different priors, a mixture of the prior distributions may be used that quite adequately represents the different beliefs held by the researchers.

Although there is enormous work on the Bayesian inference, in general the work on Bayesian analysis of randomized response techniques is limited. Researches that have been carried out on the Bayesian analysis of randomized response techniques include BarLev et al. [13], Migon and Tachibana [14], Oh [15], O’Hagan [16], Pitz [17], Unnikrishnan and Kunte [18], Winkler and Franklin [19], Adepetun and Adewara [20-23].

Winkler and Franklin [19] used beta prior distribution and showed that it results in posterior distributions as a mixture of beta distributions. They argued that it is easy to explain the mixture posterior distribution but may be difficult to work with practically. To avoid this associated difficulty; instead of analysing the mixture posterior distribution they used approximations to simplify the analysis. They also pointed out that a more appropriate prior distribution, such as truncated beta distribution, could have been used but never pursued this technique. O'Hagan [16] proposed Bayesian linear estimator for estimating the proportion of the variable of interest. He compared with the Winkler and Franklin [19] study and concluded that Bayesian linear estimators are simple and robust in the sense that least prior specification is needed but a certain price has to be paid in the situations where prior specifications can be given in details.

Bar-Lev et al. [13] argued that in view of today's computational facilities, the difficulties associated with using truncated beta distribution as prior are not strenuous. By specifying a truncated beta prior they presented a common conjugate prior structure and concluded that their approach is computationally attractive as compared to that proposed by Winkler and Franklin [19].

In this paper, we propose new stratified Bayesian estimators through mixture of alternative beta priors to analyse posterior distribution with fully specified prior information instead of approximating the posterior or taking a truncated beta prior distribution as done respectively by Winkler and Franklin [19] and Bar-Lev et. al. [13].

With Warner [1] Randomized Response Technique (RRT). The Randomized Response Technique introduced by Warner [1] has the goal of decreasing biasness in the parameter's estimates and increasing the response rate. Warner's technique consists of two complimentary questions $A$ (do you belong to stigmatized group?) and $A^{c}$ (do you not belong to stigmatized group?) to be answered on probability basis. Assuming simple random sampling with replacement the $i^{\text {th }}$ chosen respondent is asked to choose a question $\left(A\right.$ or $\left.A^{c}\right)$ and report "yes" if his/her real status tallies with chosen question and 
"no" otherwise. Denoting the probability of selecting question $A$ by $p$ and population proportion of individuals with stigmatized characteristic by $\pi$ the probability of "yes" for a particular respondent, denoted by

$\varphi$ was given as:

$P(y e s)=\varphi=p \pi+(1-p)(1-\pi)$

Solving (1) for $\pi$, we have:

$$
\pi=\frac{\varphi-(1-p)}{2 p-1}
$$

In the classical development, the maximum likelihood estimator (MLE) of $\pi$ was given as

$$
\hat{\pi}_{M L}=\frac{\hat{\varphi}-(1-p)}{2 p-1}
$$

where $\hat{\varphi}=\frac{x}{n}$ and $x$ is the number of yes responses in the sample of size $n$.

The rest of the article is organized as follows. Section 2 provides the conventional technique using Warner [1] RRT in a Bayesian framework using stratified mixture of simple beta priors followed by the proposed stratified Bayesian estimators in section 3 . Section 4 contains performance evaluation and comparison. Section 5 is the conclusion of our article.

\section{The Conventional Stratified Bayesian Technique of Estimation}

There may be different beliefs about the shape of the distribution of the parameter of the interest among different researchers. Participation of more than one researcher in a study can lead to disparities about the content of the prior information. Each may choose a different shape of the prior distribution of the parameter of interest. Sharing all of the available information among the researchers can help to reduce these disparities. In the long run, however, there may be no single values of the hyper parameters that satisfy them. In such situations, a mixture of the prior distributions may be used that quite satisfactorily represents different beliefs held by the researchers.

Assume the participation of $M$ researchers with their own beliefs about the prior distribution. By representing them with $f_{1}(\pi), f_{2}(\pi), \ldots, f_{M}(\pi)$, a mixture of prior distributions $f(\pi)=\sum_{h=1}^{M} q_{h} f_{h}(\pi)$ can be defined such that $\sum_{h=1}^{M} q_{h}=1$ where $q_{1}, q_{2}, \ldots, q_{M}$ are stratum weights associated with each prior distribution.

Suppose $h^{\text {th }}$ researcher has his belief about the prior distribution as

$f_{h}\left(\pi \mid a_{h}, b_{h}\right)=\frac{\pi^{a_{h}-1}(1-\pi)^{b_{h}-1}}{\beta\left(a_{h}, b_{h}\right)} \quad ; a_{h}, b_{h}>0, \quad h=1,2, \ldots, 4 ; 0<\pi<1$

Then a stratified mixed prior was given as

$$
f\left(\pi \mid a^{\prime} s, b^{\prime} s\right)=\sum_{h=1}^{M} q_{h}\left(\frac{\pi^{a_{h}-1}(1-\pi)^{b_{h}-1}}{\beta\left(a_{h}, b_{h}\right)}\right) ; 0<\pi<1
$$


The stratified joint density distribution of $\pi$ and $X$ was given as

$f(X, \pi)=H \sum_{h=1}^{M}\left[\left(\frac{q_{h}}{\beta\left(a_{h}, b_{h}\right)}\right) \sum_{i=0}^{x} \sum_{j=0}^{n-x}\left(\begin{array}{l}x \\ i\end{array}\right)\left(\begin{array}{c}n-x \\ j\end{array}\right) f^{n-i-j} \pi^{a_{h}+i-1}(1-\pi)^{b_{h}+j-1}\right]$

where $H=\left(\begin{array}{l}n \\ x\end{array}\right)(2 p-1)^{n} ; f=\frac{1-p}{2 p-1}$ and $x=0,1,2, \ldots n$

The stratified marginal distribution of $X$ was found by integrating the stratified joint density distribution with respect to $\pi$ over the support of 0 and 1 as

$$
f(X)=H \sum_{h=1}^{M}\left[\left(\frac{q_{h}}{\beta\left(a_{h}, b_{h}\right)}\right) \sum_{i=0}^{x} \sum_{j=0}^{n-x}\left(\begin{array}{l}
x \\
i
\end{array}\right)\left(\begin{array}{c}
n-x \\
j
\end{array}\right) f^{n-i-j} \beta\left(a_{h}+i, b_{h}+j\right)\right]
$$

Thus, the stratified posterior distribution of $\pi$ given $X$ was given as

$f(\pi \mid X)=\frac{\sum_{h=1}^{M}\left[\left(\frac{q_{h}}{\beta\left(a_{h}, b_{h}\right)}\right) \sum_{i=0}^{x} \sum_{j=0}^{n-x}\left(\begin{array}{c}x \\ i\end{array}\right)\left(\begin{array}{c}n-x \\ j\end{array}\right) f^{n-i-j} \pi^{a_{h}+i-1}(1-\pi)^{b_{h}+j-1}\right]}{\sum_{h=1}^{M}\left[\left(\frac{q_{h}}{\beta\left(a_{h}, b_{h}\right)}\right) \sum_{i=0}^{x} \sum_{j=0}^{n-x}\left(\begin{array}{c}x \\ i\end{array}\right)\left(\begin{array}{c}n-x \\ j\end{array}\right) f^{n-i-j} \beta\left(a_{h}+i, b_{h}+j\right)\right]}$

Assuming the square error loss function, the stratified Bayes estimator was given by the mean of the stratified posterior distribution of $\pi$ expressed as

$\hat{\pi}_{M W}=\frac{\sum_{h=1}^{M}\left[\left(\frac{q_{h}}{\beta\left(a_{h}, b_{h}\right)}\right) \sum_{i=0}^{x} \sum_{j=0}^{n-x}\left(\begin{array}{c}x \\ i\end{array}\right)\left(\begin{array}{c}n-x \\ j\end{array}\right) f^{n-i-j} \beta\left(a_{h}+i+1, b_{h}+j\right)\right]}{\sum_{h=1}^{M}\left[\left(\frac{q_{h}}{\beta\left(a_{h}, b_{h}\right)}\right) \sum_{i=0}^{x} \sum_{j=0}^{n-x}\left(\begin{array}{c}x \\ i\end{array}\right)\left(\begin{array}{c}n-x \\ j\end{array}\right) f^{n-i-j} \beta\left(a_{h}+i, b_{h}+j\right)\right]}$

Consequently, the bias as well as the mean square error of the stratified Bayes estimator was given as

$\operatorname{Bias}\left(\hat{\pi}_{M W}\right)=E\left(\hat{\pi}_{M W}\right)-\pi$

$\operatorname{MSE}\left(\hat{\pi}_{M W}\right)=\sum_{x=0}^{n}\left(\hat{\pi}_{M W}-\pi\right)^{2}\left(\begin{array}{l}n \\ x\end{array}\right) \varphi^{x}(1-\varphi)^{n-x}$

\section{The Proposed Stratified Bayesian Techniques of Estimation}

Following study of the performance of the Bayesian estimator developed by Hussain et al. [24] in a stratified random sampling environment, we propose alternative stratified Bayesian estimators assuming both Kumaraswamy (KUMA) and the generalised (GLS) beta priors respectively.

\subsection{Estimation of $\pi$ using Kumaraswamy prior}

Assume $h^{\text {th }}$ researcher has his belief about the prior distribution as $f\left(\pi \mid b_{h}, c_{h}\right)=b_{h} c_{h} \pi^{c_{h}-1}\left(1-\pi^{c_{h}}\right)^{b_{h}-1} \quad ; b_{h}, c_{h}>0, h=1,2, \ldots, 4 ; 0<\pi<1$ 
Then a stratified mixed prior can then be expressed as

$$
f\left(\pi \mid b^{\prime} s, c^{\prime} s\right)=\sum_{h=1}^{M} q_{h} b_{h} c_{h} \pi^{c_{h}-1}\left(1-\pi^{c_{h}}\right)^{b_{h}-1} \quad ; 0<\pi<1
$$

Accordingly, the stratified joint density distribution of $X$ and $\pi$ is

$$
F(X, \pi)=H \sum_{h=1}^{M}\left[A_{h} \sum_{i=0}^{x} \sum_{j=0}^{n-x}\left(\begin{array}{l}
x \\
i
\end{array}\right)\left(\begin{array}{c}
n-x \\
j
\end{array}\right) f^{n-i-j} \pi^{c_{h}-1}\left(1-\pi^{c_{h}}\right)^{b_{h}-1} \pi^{i}(1-\pi)^{j}\right]
$$

For simplicity purpose, this can be re-written as

$$
F(X, \pi)=H \sum_{h=1}^{M}\left[A_{h} \sum_{i=0}^{x} \sum_{j=0}^{n-x} \sum_{k=0}^{b_{h}-1}(-1)^{k}\left(\begin{array}{c}
x \\
i
\end{array}\right)\left(\begin{array}{c}
n-x \\
j
\end{array}\right)\left(\begin{array}{c}
b_{h}-1 \\
k
\end{array}\right) f^{n-i-j} \pi^{c_{h}+c_{h} k+i-1}(1-\pi)^{j}\right]
$$

where $A_{h}=q_{h} b_{h} c_{h}$

The stratified marginal distribution of $X$ after integrating the stratified joint density distribution above with respect to $\pi$ between interval 0 and 1 is

$$
F(X)=H \sum_{h=1}^{M}\left[A_{h} \sum_{i=0}^{x} \sum_{j=0}^{n-x} \sum_{k=0}^{b_{h}-1}(-1)^{k}\left(\begin{array}{c}
x \\
i
\end{array}\right)\left(\begin{array}{c}
n-x \\
j
\end{array}\right)\left(\begin{array}{c}
b_{h}-1 \\
k
\end{array}\right) f^{n-i-j} \beta\left(c_{h} k+c_{h}+i, j+1\right)\right]
$$

The stratified posterior distribution of $\pi$ given $\mathrm{X}$ is

$$
\begin{aligned}
& F(\pi \mid X)=\frac{F(X, \pi)}{F(X)} \\
& =\frac{\sum_{h=1}^{M}\left[A_{h} \sum_{i=0}^{x} \sum_{j=0}^{n-x} \sum_{k=0}^{b_{h}-1}(-1)^{k}\left(\begin{array}{c}
x \\
i
\end{array}\right)\left(\begin{array}{c}
n-x \\
j
\end{array}\right)\left(\begin{array}{c}
b_{h-1} \\
k
\end{array}\right) f^{n-i-j} \pi^{c_{h} k+c_{h}-1+i}(1-\pi)^{j}\right]}{\sum_{h=1}^{M}\left[A_{h} \sum_{i=0}^{x} \sum_{j=0}^{n-x} \sum_{k=0}^{b_{h}-1}(-1)^{k}\left(\begin{array}{c}
x \\
i
\end{array}\right)\left(\begin{array}{c}
n-x \\
j
\end{array}\right)\left(\begin{array}{c}
b_{h}-1 \\
k
\end{array}\right) f^{n-i-j} \beta\left(c_{h} k+c_{h}+i, j+1\right)\right]}
\end{aligned}
$$

Under square error loss, the proposed stratified Bayesian estimator of $\pi$ assuming Kumaraswamy prior is

$\hat{\pi}_{M K}=\frac{\sum_{h=1}^{M}\left[A_{h} \sum_{i=0}^{x} \sum_{j=0}^{n-x} \sum_{k=0}^{b_{h}-1}(-1)^{k}\left(\begin{array}{c}x \\ i\end{array}\right)\left(\begin{array}{c}n-x \\ j\end{array}\right)\left(\begin{array}{c}b_{h}-1 \\ k\end{array}\right) f^{n-i-j} \beta\left(c_{h} k+c_{h}+i+1, j+1\right)\right]}{\sum_{h=1}^{M}\left[A_{h} \sum_{i=0}^{x} \sum_{j=0}^{n-x} \sum_{k=0}^{b_{h}-1}(-1)^{k}\left(\begin{array}{c}x \\ i\end{array}\right)\left(\begin{array}{c}n-x \\ j\end{array}\right)\left(\begin{array}{c}b_{h}-1 \\ k\end{array}\right) f^{n-i-j} \beta\left(c_{h} k+c_{h}+i, j+1\right)\right]}$

The bias as well as the mean square error of $\hat{\pi}_{M K}$ is given as

$$
\begin{aligned}
& \operatorname{Bias}\left(\hat{\pi}_{M K}\right)=E\left(\hat{\pi}_{M K}\right)-\pi \\
& \operatorname{MSE}\left(\hat{\pi}_{M K}\right)=\sum_{x=0}^{n}\left(\hat{\pi}_{M K}-\pi\right)^{2}\left(\begin{array}{l}
n \\
x
\end{array}\right) \varphi^{x}(1-\varphi)^{n-x}
\end{aligned}
$$

\subsection{Estimation of $\pi$ using the generalised beta prior}

Let $\mathrm{h}^{\text {th }}$ researcher has his belief about the prior distribution as 
$f_{h}\left(\pi \mid a_{h}, b_{h}, c_{h}\right)=\frac{c_{h} \pi^{a_{h} c_{h}-1}\left(1-\pi^{c_{h}}\right)^{b_{h}-1}}{\beta\left(a_{h}, b_{h}\right)} ; a_{h}, b_{h}, c_{h}>0, h=1,2, \ldots, 4 ; 0<\pi<1$

We can, therefore, write our mixed prior as

$$
f\left(\pi \mid a^{\prime} s, b^{\prime} s, c^{\prime} s\right)=\sum_{h=1}^{M} q_{h} \frac{c_{h} \pi^{a_{h} c_{h-1}}\left(1-\pi^{c_{h}}\right)^{b_{h}-1}}{\beta\left(a_{h}, b_{h}\right)} ; 0<\pi<1
$$

Consequently, the stratified joint density distribution of $X$ and $\pi$ is written as

$$
\begin{aligned}
& F_{o}(X, \pi)=H \sum_{h=1}^{M}\left[D_{h} \sum_{i=0}^{x} \sum_{j=0}^{n-x} \sum_{k=0}^{b_{h}-1}(-1)^{k}\left(\begin{array}{l}
x \\
i
\end{array}\right)\left(\begin{array}{c}
n-x \\
j
\end{array}\right)\left(\begin{array}{c}
b_{h}-1 \\
k
\end{array}\right) f^{n-i-j} \pi^{c_{h}\left(k+a_{h}\right)+i-1}(1\right. \\
& \left.-\pi)^{j}\right]
\end{aligned}
$$

where $D_{h}=\frac{q_{h} c_{h}}{\beta\left(a_{h}, b_{h}\right)}$

The stratified marginal distribution of $X$ is thus

$$
\begin{gathered}
F_{o}(X)=H \sum_{h=1}^{M}\left[\begin{array}{c}
D_{h} \\
\sum_{i=0}^{x} \sum_{j=0}^{n-x} \sum_{k=0}^{b_{h}-1}(-1)^{k}\left(\begin{array}{c}
x \\
i
\end{array}\right)\left(\begin{array}{c}
n-x \\
j
\end{array}\right)\left(\begin{array}{c}
b_{h}-1 \\
k
\end{array}\right) f^{n-i-j} \beta\left(c_{h}\left(k+a_{h}\right)+i, j\right. \\
+1)
\end{array}\right]
\end{gathered}
$$

As a result, the stratified posterior distribution of $\pi$ given $X$ is

$F_{o}(\pi \mid X)$

$$
=\frac{\sum_{h=1}^{M}\left[D_{h} \sum_{i=0}^{x} \sum_{j=0}^{n-x} \sum_{k=0}^{b_{h}-1}(-1)^{k}\left(\begin{array}{c}
x \\
i
\end{array}\right)\left(\begin{array}{c}
n-x \\
j
\end{array}\right)\left(\begin{array}{c}
b_{h}-1 \\
k
\end{array}\right) f^{n-i-j} \pi^{c_{h}\left(k+a_{h}\right)+i-1}(1-\pi)^{j}\right]}{\sum_{h=1}^{M}\left[D_{h} \sum_{i=0}^{x} \sum_{j=0}^{n-x} \sum_{k=0}^{b_{h}-1}(-1)^{k}\left(\begin{array}{c}
x \\
i
\end{array}\right)\left(\begin{array}{c}
n-x \\
j
\end{array}\right)\left(\begin{array}{c}
b_{h}-1 \\
k
\end{array}\right) f^{n-i-j} \beta\left(c_{h}\left(k+a_{h}\right)+i, j+1\right)\right]}
$$

Under the square error loss, the proposed stratified Bayesian estimator assuming the generalised beta prior is obtained as

$$
\left.\hat{\pi}_{M G}=\frac{\sum_{h=1}^{M}\left[D_{h} \sum_{i=0}^{x} \sum_{j=0}^{n-x} \sum_{k=0}^{b_{h}-1}(-1)^{k}\left(\begin{array}{c}
x \\
i
\end{array}\right)\left(\begin{array}{c}
n-x \\
j
\end{array}\right)\left(\begin{array}{c}
b_{h}-1 \\
k
\end{array}\right) f^{n-i-j} \beta\left(c_{h}\left(k+a_{h}\right)+i+1, j+1\right)\right]}{\sum_{h=1}^{M}\left[D_{h} \sum_{i=0}^{x} \sum_{j=0}^{n-x} \sum_{k=0}^{b_{h}-1}(-1)^{k}\left(\begin{array}{c}
x \\
i
\end{array}\right)\left(\begin{array}{c}
n-x \\
j
\end{array}\right)\left(\begin{array}{c}
b_{h}-1 \\
k
\end{array}\right) f^{n-i-j} \beta\left(c_{h}\left(k+a_{h}\right)+i, j+1\right)\right]} 25\right)
$$

The bias and the mean square error (MSE) of $\hat{\pi}_{M G}$ are defined respectively as

$$
\begin{aligned}
& \operatorname{Bias}\left(\hat{\pi}_{M G}\right)=E\left(\hat{\pi}_{M G}\right)-\pi \\
& \operatorname{MSE}\left(\hat{\pi}_{M G}\right)=\sum_{x=0}^{n}\left(\hat{\pi}_{M G}-\pi\right)^{2}\left(\begin{array}{l}
n \\
x
\end{array}\right) \varphi^{x}(1-\varphi)^{n-x}
\end{aligned}
$$

Remark: In this study, we limit the number of our researchers to 4 , that is $M=4$. 


\section{Performance Evaluation and Comparisons}

Before moving towards formal comparisons of the proposed stratified Bayesian estimators with that of Hussain et al. [24], we point out some computational complexities associated with our study. From derivations in this work, it is clear that both the conventional and the proposed stratified Bayesian estimators involve enormous computation especially when the sample size and/or the number of "yes" responses are/is large. To deal with this computational complexity, we have written a program in $\mathrm{R}$ software (this program is available with the corresponding author).

We validated the performance of our proposed stratified Bayesian estimators in capturing responses from respondents through the life data obtained from the administered survey questionnaires on induced abortion under the same values of parameters in the estimators using sample sizes 25, 100 and 250 respectively. Few results in Tables were presented to reduce spaces. Concise evaluation performances of the proposed Bayesian estimators in a stratified random sampling scenario were also stated in the form of comments after tables for each sample size considered in this study respectively.

Table 1. Mean square errors for Warner [1] RRT at $n=25, x=11, p=0.6$.

\begin{tabular}{llll}
\hline$\pi$ & MSE Beta & MSE Kuma & MSE Gls \\
\hline 0.1 & $1.12 \mathrm{E}-07$ & $1.11 \mathrm{E}-06$ & $1.50 \mathrm{E}-06$ \\
0.2 & $3.28 \mathrm{E}-08$ & $8.12 \mathrm{E}-07$ & $1.15 \mathrm{E}-06$ \\
0.3 & $7.55 \mathrm{E}-10$ & $5.58 \mathrm{E}-07$ & $8.43 \mathrm{E}-07$ \\
0.4 & $1.59 \mathrm{E}-08$ & $3.52 \mathrm{E}-07$ & $5.84 \mathrm{E}-07$ \\
0.5 & $7.83 \mathrm{E}-08$ & $1.94 \mathrm{E}-07$ & $3.73 \mathrm{E}-07$ \\
0.6 & $1.88 \mathrm{E}-07$ & $8.20 \mathrm{E}-08$ & $2.09 \mathrm{E}-07$ \\
0.7 & $3.45 \mathrm{E}-07$ & $1.76 \mathrm{E}-08$ & $9.21 \mathrm{E}-08$ \\
0.8 & $5.48 \mathrm{E}-07$ & $4.34 \mathrm{E}-10$ & $2.25 \mathrm{E}-08$ \\
0.9 & $8.00 \mathrm{E}-07$ & $3.04 \mathrm{E}-08$ & $1.37 \mathrm{E}-11$ \\
\hline
\end{tabular}

Table 2. Absolute bias for Warner [1] RRT at $n=25, x=11, p=0.6$.

\begin{tabular}{llll}
\hline$\pi$ & |BIAS|Beta & |BIAS|Kuma & BIAS|Gls \\
\hline 0.1 & 0.217891 & 0.686433 & 0.797589 \\
0.2 & 0.117891 & 0.586433 & 0.697589 \\
0.3 & 0.017891 & 0.486433 & 0.597589 \\
0.4 & 0.082109 & 0.386433 & 0.497589 \\
0.5 & 0.182109 & 0.286433 & 0.397589 \\
0.6 & 0.282109 & 0.186433 & 0.297589 \\
0.7 & 0.382109 & 0.086433 & 0.197589 \\
0.8 & 0.482109 & 0.013567 & 0.097589 \\
0.9 & 0.582109 & 0.113567 & 0.002411 \\
\hline
\end{tabular}

Table 3. Mean square errors for Warner [1] RRT at $n=25, x=11, p=0.7$.

\begin{tabular}{llll}
\hline$\pi$ & MSE Beta & MSE Kuma & MSE Gls \\
\hline 0.1 & $1.25 \mathrm{E}-07$ & $1.01 \mathrm{E}-06$ & $1.45 \mathrm{E}-06$ \\
0.2 & $3.99 \mathrm{E}-08$ & $7.22 \mathrm{E}-07$ & $1.10 \mathrm{E}-06$ \\
0.3 & $2.13 \mathrm{E}-09$ & $4.85 \mathrm{E}-07$ & $8.01 \mathrm{E}-07$
\end{tabular}




\begin{tabular}{llll}
$\pi$ & MSE Beta & MSE Kuma & MSE Gls \\
\hline 0.4 & $1.15 \mathrm{E}-08$ & $2.94 \mathrm{E}-07$ & $5.50 \mathrm{E}-07$ \\
0.5 & $6.82 \mathrm{E}-08$ & $1.51 \mathrm{E}-07$ & $3.46 \mathrm{E}-07$ \\
0.6 & $1.72 \mathrm{E}-07$ & $5.54 \mathrm{E}-08$ & $1.89 \mathrm{E}-07$ \\
0.7 & $3.23 \mathrm{E}-07$ & $6.67 \mathrm{E}-09$ & $7.88 \mathrm{E}-08$ \\
0.8 & $5.21 \mathrm{E}-07$ & $5.18 \mathrm{E}-09$ & $1.62 \mathrm{E}-08$ \\
0.9 & $7.67 \mathrm{E}-07$ & $5.09 \mathrm{E}-08$ & $7.02 \mathrm{E}-10$ \\
\hline
\end{tabular}

Table 4. Absolute bias for Warner [1] RRT at $n=25, x=11, p=0.7$.

\begin{tabular}{llll}
\hline$\pi$ & $\mid$ BIAS|Beta & $\mid$ BIAS $\mid$ Kuma & $\mid$ BIAS $\mid$ Gls \\
\hline 0.1 & 0.230050 & 0.653170 & 0.782749 \\
0.2 & 0.130050 & 0.553170 & 0.682749 \\
0.3 & 0.030050 & 0.453170 & 0.582749 \\
0.4 & 0.069950 & 0.353170 & 0.482749 \\
0.5 & 0.169950 & 0.253170 & 0.382749 \\
0.6 & 0.269950 & 0.153170 & 0.282749 \\
0.7 & 0.369950 & 0.053170 & 0.182749 \\
0.8 & 0.469950 & 0.046830 & 0.082749 \\
0.9 & 0.569950 & 0.146830 & 0.017251 \\
\hline
\end{tabular}

Comment: From tables 1 to 4 , when $n=25, x=11, P=0.6$ and 0.7 , the proposed stratified Bayesian estimators are more efficient in capturing sensitive information than the conventional one when $\pi>0.5$. The proposed stratified generalised beta estimator is optimally efficient while compared with other estimators as $\pi$ approaches one.

Table 5. Mean square errors for Warner [1] RRT at $n=100, x=43, p=0.6$.

\begin{tabular}{llll}
\hline$\pi$ & MSE Beta & MSE Kuma & MSE Gls \\
\hline 0.1 & $5.36 \mathrm{E}-26$ & $6.16 \mathrm{E}-25$ & $9.43 \mathrm{E}-25$ \\
0.2 & $1.12 \mathrm{E}-26$ & $4.35 \mathrm{E}-25$ & $7.15 \mathrm{E}-25$ \\
0.3 & $3.80 \mathrm{E}-28$ & $2.85 \mathrm{E}-25$ & $5.19 \mathrm{E}-25$ \\
0.4 & $2.10 \mathrm{E}-26$ & $1.67 \mathrm{E}-25$ & $3.54 \mathrm{E}-25$ \\
0.5 & $7.32 \mathrm{E}-26$ & $8.00 \mathrm{E}-26$ & $2.20 \mathrm{E}-25$ \\
0.6 & $1.57 \mathrm{E}-25$ & $2.47 \mathrm{E}-26$ & $1.18 \mathrm{E}-25$ \\
0.7 & $2.72 \mathrm{E}-25$ & $1.01 \mathrm{E}-27$ & $4.77 \mathrm{E}-26$ \\
0.8 & $4.19 \mathrm{E}-25$ & $8.78 \mathrm{E}-27$ & $8.62 \mathrm{E}-27$ \\
0.9 & $5.97 \mathrm{E}-25$ & $4.80 \mathrm{E}-26$ & $1.07 \mathrm{E}-27$ \\
\hline
\end{tabular}

Table 6. Absolute bias for Warner [1] RRT at $n=100, x=43, p=0.6$.

\begin{tabular}{llll}
\hline$\pi$ & $\mid$ BIAS $\mid$ Beta & $\mid$ BIAS $\mid$ Kuma & $\mid$ BIAS $\mid$ Gls \\
\hline 0.1 & 0.184457 & 0.625352 & 0.773992 \\
0.2 & 0.084457 & 0.525352 & 0.673992 \\
0.3 & 0.015543 & 0.425352 & 0.573992 \\
0.4 & 0.115543 & 0.325352 & 0.473992 \\
0.5 & 0.215543 & 0.225352 & 0.373992 \\
0.6 & 0.315543 & 0.125352 & 0.273992 \\
0.7 & 0.415543 & 0.025352 & 0.173992 \\
0.8 & 0.515543 & 0.074648 & 0.073992 \\
0.9 & 0.615543 & 0.174648 & 0.026008 \\
\hline
\end{tabular}


Table 7. Mean square errors for Warner [1] RRT at $n=100, x=43, p=0.7$.

\begin{tabular}{llll}
\hline$\pi$ & MSE Beta & MSE Kuma & MSE Gls \\
\hline 0.1 & $7.75 \mathrm{E}-26$ & $3.85 \mathrm{E}-25$ & $6.30 \mathrm{E}-25$ \\
0.2 & $2.34 \mathrm{E}-26$ & $2.45 \mathrm{E}-25$ & $4.47 \mathrm{E}-25$ \\
0.3 & $7.49 \mathrm{E}-28$ & $1.36 \mathrm{E}-25$ & $2.95 \mathrm{E}-25$ \\
0.4 & $9.63 \mathrm{E}-27$ & $5.95 \mathrm{E}-26$ & $1.74 \mathrm{E}-25$ \\
0.5 & $5.00 \mathrm{E}-26$ & $1.40 \mathrm{E}-26$ & $8.52 \mathrm{E}-26$ \\
0.6 & $1.22 \mathrm{E}-25$ & $5.03 \mathrm{E}-29$ & $2.77 \mathrm{E}-26$ \\
0.7 & $2.25 \mathrm{E}-25$ & $1.76 \mathrm{E}-26$ & $1.67 \mathrm{E}-27$ \\
0.8 & $3.60 \mathrm{E}-25$ & $6.66 \mathrm{E}-26$ & $7.16 \mathrm{E}-27$ \\
0.9 & $5.26 \mathrm{E}-25$ & $1.47 \mathrm{E}-25$ & $4.41 \mathrm{E}-26$ \\
\hline
\end{tabular}

Table 8. Absolute bias for Warner [1] RRT at $n=100, x=43, p=0.7$.

\begin{tabular}{llll}
\hline$\pi$ & $\mid$ BIAS|Beta & $\mid$ BIAS $\mid$ Kuma & $\mid$ BIAS| Gls \\
\hline 0.1 & 0.221811 & 0.494350 & 0.632591 \\
0.2 & 0.121811 & 0.394350 & 0.532591 \\
0.3 & 0.021811 & 0.294350 & 0.432591 \\
0.4 & 0.078189 & 0.194350 & 0.332591 \\
0.5 & 0.178189 & 0.094350 & 0.232591 \\
0.6 & 0.278189 & 0.005650 & 0.132591 \\
0.7 & 0.378189 & 0.105650 & 0.032591 \\
0.8 & 0.478189 & 0.205650 & 0.067409 \\
0.9 & 0.578189 & 0.305650 & 0.167409 \\
\hline
\end{tabular}

Comment: From tables 5 to 8 , when $n=100, x=43, p=0.6$ and 0.7 , the proposed stratified Bayesian estimators are more efficient in capturing sensitive information from respondents than the conventional stratified simple beta estimator proposed by Hussain et al. [24] when $\pi>0.5$. The proposed stratified generalised beta estimator is optimally efficient as $\pi$ approaches one.

Table 9. Mean square errors for Warner [1] RRT at $n=250, x=106, p=0.6$.

\begin{tabular}{llll}
\hline$\pi$ & MSE Beta & MSE Kuma & MSE Gls \\
\hline 0.1 & $9.18 \mathrm{E}-62$ & $1.09 \mathrm{E}-60$ & $2.09 \mathrm{E}-60$ \\
0.2 & $6.91 \mathrm{E}-63$ & $6.80 \mathrm{E}-61$ & $1.50 \mathrm{E}-60$ \\
0.3 & $1.87 \mathrm{E}-62$ & $3.66 \mathrm{E}-61$ & $1.01 \mathrm{E}-60$ \\
0.4 & $1.27 \mathrm{E}-61$ & $1.48 \mathrm{E}-61$ & $6.18 \mathrm{E}-61$ \\
0.5 & $3.32 \mathrm{E}-61$ & $2.72 \mathrm{E}-62$ & $3.20 \mathrm{E}-61$ \\
0.6 & $6.34 \mathrm{E}-61$ & $3.00 \mathrm{E}-63$ & $1.20 \mathrm{E}-61$ \\
0.7 & $1.03 \mathrm{E}-60$ & $7.54 \mathrm{E}-62$ & $1.60 \mathrm{E}-62$ \\
0.8 & $1.53 \mathrm{E}-60$ & $2.45 \mathrm{E}-61$ & $8.74 \mathrm{E}-63$ \\
0.9 & $2.12 \mathrm{E}-60$ & $5.10 \mathrm{E}-61$ & $9.82 \mathrm{E}-62$ \\
\hline
\end{tabular}

Table 10. Absolute bias for Warner [1] RRT at $n=250, x=106, p=0.6$.

\begin{tabular}{llll}
\hline$\pi$ & $\mid$ BIAS|Beta & $\mid$ BIAS $\mid$ Kuma & $\mid$ BIAS| Gls \\
\hline 0.1 & 0.137809 & 0.475069 & 0.657476 \\
0.2 & 0.037809 & 0.375069 & 0.557476 \\
0.3 & 0.062191 & 0.275069 & 0.457476
\end{tabular}




\begin{tabular}{llll}
$\pi$ & $\mid$ BIAS $\mid$ Beta & $\mid$ BIAS $\mid$ Kuma & $\mid$ BIAS| Gls \\
\hline 0.4 & 0.162191 & 0.175069 & 0.357476 \\
0.5 & 0.262191 & 0.075069 & 0.257476 \\
0.6 & 0.362191 & 0.024931 & 0.157476 \\
0.7 & 0.462191 & 0.124931 & 0.057476 \\
0.8 & 0.562191 & 0.224931 & 0.042524 \\
0.9 & 0.662191 & 0.324931 & 0.142524 \\
\hline
\end{tabular}

Table 11. Mean square errors for Warner [1] RRT at $n=250, x=106, p=0.7$.

\begin{tabular}{llll}
\hline$\pi$ & MSE Beta & MSE Kuma & MSE Gls \\
\hline 0.1 & $2.15 \mathrm{E}-61$ & $6.09 \mathrm{E}-61$ & $9.12 \mathrm{E}-61$ \\
0.2 & $5.94 \mathrm{E}-62$ & $3.14 \mathrm{E}-61$ & $5.40 \mathrm{E}-61$ \\
0.3 & $5.66 \mathrm{E}-64$ & $1.16 \mathrm{E}-61$ & $2.65 \mathrm{E}-61$ \\
0.4 & $3.84 \mathrm{E}-62$ & $1.45 \mathrm{E}-62$ & $8.72 \mathrm{E}-62$ \\
0.5 & $1.73 \mathrm{E}-61$ & $9.85 \mathrm{E}-63$ & $5.69 \mathrm{E}-63$ \\
0.6 & $4.04 \mathrm{E}-61$ & $1.02 \mathrm{E}-61$ & $2.09 \mathrm{E}-62$ \\
0.7 & $7.32 \mathrm{E}-61$ & $2.90 \mathrm{E}-61$ & $1.33 \mathrm{E}-61$ \\
0.8 & $1.16 \mathrm{E}-60$ & $5.76 \mathrm{E}-61$ & $3.41 \mathrm{E}-61$ \\
0.9 & $1.68 \mathrm{E}-60$ & $9.58 \mathrm{E}-61$ & $6.46 \mathrm{E}-61$ \\
\hline
\end{tabular}

Table 12. Absolute bias for Warner [1] RRT at $n=250, x=106, p=0.7$.

\begin{tabular}{llll}
\hline$\pi$ & $\mid$ BIAS $\mid$ Beta & $\mid$ BIAS $\mid$ Kuma & $\mid$ BIAS $\mid$ Gls \\
\hline 0.1 & 0.210818 & 0.354850 & 0.434300 \\
0.2 & 0.110818 & 0.254850 & 0.334300 \\
0.3 & 0.010818 & 0.154850 & 0.234300 \\
0.4 & 0.089182 & 0.054850 & 0.134300 \\
0.5 & 0.189182 & 0.045150 & 0.034300 \\
0.6 & 0.289182 & 0.145150 & 0.065700 \\
0.7 & 0.389182 & 0.245150 & 0.165700 \\
0.8 & 0.489182 & 0.345150 & 0.265700 \\
0.9 & 0.589182 & 0.445150 & 0.365700 \\
\hline
\end{tabular}

Comment: From tables 9 to 12 , when $n=250, x=106, p=0.6$ and 0.7 , the proposed stratified Bayesian estimators are better than the existing one in capturing sensitive information from respondents when $\pi>0.4$. The proposed Bayesian estimator which assumed stratified generalised beta prior is the best as $\pi$ tends to unity.

\section{Conclusion}

This article has taken up the issue of estimating proportion of a stigmatized characteristic using Randomized Response Technique in Bayesian setting under stratified random sampling procedure. To quantify prior information, we have considered completely specified mixture priors in the form of Beta distribution(s). The proposed stratified Bayesian estimators have been evaluated based on absolute bias and mean squared error and their comparisons have been made with the corresponding existing stratified simple beta estimator proposed by Hussain et al. [24]. It was observed that for the practicable 
choices of the design parameter $p$, the proposed stratified Bayesian estimators perform reasonably better in capturing information from respondents in survey which asks sensitive questions than the existing one over a wide range of population proportion $p$. It has also been observed that Bayesian estimators are bounded by 0 and 1 even in case of extreme realization of "yes" responses under the stratified random sampling design in mixture prior scenarios.

Finally, it can be concluded that whenever prior information about the likely values of the parameter of interest is available, we should opt for Bayesian approach ignoring the computational difficulty in the presence of today's computational facilities.

\section{References}

1. S. L. Warner, J. Am. Statist. Assoc. 60, 63 (1965). https://doi.org/10.1080/01621459.1965.10480775

2. B. Greenberg, A. Abul-Ela, W. Simmons, and D. Horvitz, J. Am. Stat. Assoc. 64, 529 (1969). https://doi.org/10.1080/01621459.1969.10501009

3. J. J. Moors, J. Am. Stat. Assoc. 66, 627 (1971). https://doi.org/10.1080/01621459.1971.10482320

4. N. S. Mangat, J. Royal Stat. Soc. Ser. B 56, 93 (1994).

5. S. Singh, S. Horn, and S. Chowdhuri, Australian \& New Zealand J. Stat. 40, 291 (1998). https://doi.org/10.1111/1467-842X.00033

6. T. C. Christofides, Metrika 57,195 (2003). https://doi.org/10.1007/s001840200216

7. J. M. Kim and D. W. Warde, J. Stat. Plan. Infer. 120,155 (2004). https://doi.org/10.1016/S0378-3758(02)00500-1

8. J. M. Kim and D. W. Warde, J. Stat. Plan. Infer. 133, 211 (2005). https://doi.org/10.1016/j.jspi.2004.03.011

9. A. Chaudhuri and R. Mukerjee (Marcel Decker, New York, 1998).

10. D. Tracy and N. Mangat, J. Appl. Stat. Sci., 4, 533 (1996).

11. F. B. Adebola and A. O. Adepetun, Int. J. Math. Theory Modeling 2, 61 (2012).

12. A. O. Adepetun and F. B. Adebola, Int. J. Math. Theory Modeling 4, 58 (2014).

13. S. K. Bar-Lev, E. Bobovich, and B. Boukai, Test 12, 101 (2003). https://doi.org/10.1007/BF02595813

14. H. Migon and V. Tachibana, Computational Statistics Data Anal. 24, 401 (1997). https://doi.org/10.1016/S0167-9473(96)00075-8

15. M. Oh, J. Korean Stat. Soc. 23, 463 (1994).

16. O’ Hagan, J. Am. Stat. Assoc. 82, 580 (1987). https://doi.org/10.1080/01621459.1987.10478469

17. G. Pitz, J. Psycho. Bull. 87, 209 (1980).

18. N. Unnikrishnan and S. Kunte, Sankhya Ser. B 61, 422 (1999).

19. R. Winkler and L. Franklin, J. Am. Stat. Assoc. 74, 207 (1979). https://doi.org/10.1080/01621459.1979.10481639

20. A. O. Adepetun and A. A. Adewara, Am. J. of Comput. Appl. Math. 4, 130 (2014).

21. A. O. Adepetun and A. A. Adewara, Int. J. Scient. Eng. Res. 6, 1634 (2015).

22. A. O. Adepetun and A. A. Adewara, Punjab Univ. J. Math. 48, 29 (2016).

23. A. O. Adepetun and A. A. Adewara, J. Sci. Res. 9, 13 (2017). https://doi.org/10.3329/jsr.v1i1.27943

24. Z. Hussain, J. Shabbir, and R. Muhammad, Commun. Statistics, Simulation Comput. 40, 147 (2010). https://doi.org/10.1080/03610918.2010.532897 